\section{Antidiabetic Activity of Green Tea (Thea sinensis L.) in Genetically Type 2 Diabetic Mice}

\author{
Toshihiro Miura, ${ }^{*}, a$ Tomoko Koike, ${ }^{a}$ \\ and Torao Ishida ${ }^{b}$
}

${ }^{a}$ Department of Clinical Nutrition, and ${ }^{b} \mathrm{Hi}$-tech Research Center, Suzuka University of Medical Science, 1001-1 Kishioka, Suzuka, Mie 510-0293, Japan

(Received April 21, 2005; Accepted August 28, 2005)

The antidiabetic activity of Thea sinensis was investigated in KK-Ay mice, an animal model of genetically type 2 diabetes with hyperinsulinemia. The water extract of Thea sinensis (green tea cold ext) (100 $\mathrm{mg} / \mathrm{kg}$ body weight) reduced the blood glucose of KK-Ay mice 4 and 8 weeks after repeated administration and tended to decrease the plasma insulin level of KK-Ay mice under similar conditions. However, green tea cold ext did not affect blood glucose levels in normal mice. Green tea cold ext decreased blood glucose levels in an insulin tolerance test. These results suggest that the antidiabetic activity of green tea cold ext is derived, at least in part, from a decrease in plasma insulin, due to decreased insulin resistance.

Key words — green tea, antidiabetic activity, KKAy mice, Thea sinensis, insulin resistance

\section{INTRODUCTION}

Insulin resistance in peripheral tissues, together with the impairment of glucose-induced insulin secretion from pancreatic beta cells, is known as one of the major pathogenic factors of type 2 diabetes. Almost all cases of diabetes mellitus are type 2 . Therapeutic agents to stimulate insulin secretion (for example, sulfonylureas) have been used to treat type 2 diabetic patients. However, from the first stage of this condition, diet and exercise therapy are prescribed.

Thea sinensis (T. sinensis), which contains catechin, has been used in Japan as a traditional luxury.

\footnotetext{
*To whom correspondence should be addressed: Department of Clinical Nutrition, Suzuka University of Medical Science, 10011 Kishioka, Suzuka, Mie 510-0293, Japan. Tel.: +81-593-838991; Fax: +81-593-83-9666; E-mail: miura@ suzuka-u.ac.jp
}

It is known that catechin improves hyperglycemia. ${ }^{1)}$ Shimizu et al. reported on the hypoglycemic activity of Japanese tea in normal and streptozotocin-induced diabetic rats, and one of the hypoglycemic components in normal rats was identified as a polysaccharide. ${ }^{2)}$ However, few studies have been performed in type 2 diabetes models. In the present study, we examined the effects of $T$. sinensis on blood glucose levels in type 2 diabetic mice, along with the effects of insulin resistance, to clarify the antidiabetic mechanism. It is known that polysaccharides improve glucose metabolism and that they are not heat resistant. ${ }^{2)}$ Therefore we used the cold extract of green tea.

\section{MATERIALS AND METHODS}

T. sinensis provided by JA Mie Keizairen Hokusei-cha Center Co., Ltd. (Suzuka, Mie, Japan) as used in the present experiment. One hundred grams of this was extracted with 0.71 of water $\left(20^{\circ} \mathrm{C}\right.$, $1 \mathrm{hr}$, twice). After filtration (filter paper no. 51B), the water extracts were lyophilized (green tea cold ext) and stored at room temperature until use. The yield was $12.9 \%$ of cold ext.

Animals — Adult male ddY mice (SLC, Shizuoka, Japan) weighing 22-25 g and KK-Ay mice (Clea, Tokyo, Japan), 5 weeks old, were used. KKAy mice with blood glucose levels $>250 \mathrm{mg} / 100 \mathrm{ml}$ (ad libitum) were considered to be diabetic and used in this study. The mice were housed in an air-conditioned room at $22 \pm 2^{\circ} \mathrm{C}$ with a 12-hr light-12-hr dark cycle (light: 09:00 am to 21:00). The animals were kept in an experimental animal room for 7 days with free access to food (CE-2, Clea) and water (tap water). Blood samples were drawn from the cavernous sinus with a capillary to determine blood glucose levels under nonanesthetized conditions. Green tea extracts were dissolved in distilled water. The distilled water was administered to the control mice. The studies were started at 10:00-11:00, and blood samples after repeated administration of extract were taken at 10:00-11:00. The extract was administered orally on a compulsory basis (repeated administration, once a day). Animals were treated in accodance with the Guidelines for the Care and Use of Laboratory Animals (Prime Minister's Office no. 6, 1980). Insulin Tolerance Test — Insulin tolerance tests were performed at the end of the repeated administration. After overnight (18-hr) fasting, insulin $(0.5 \mathrm{U} / \mathrm{kg}$ body weight) solution was administered 


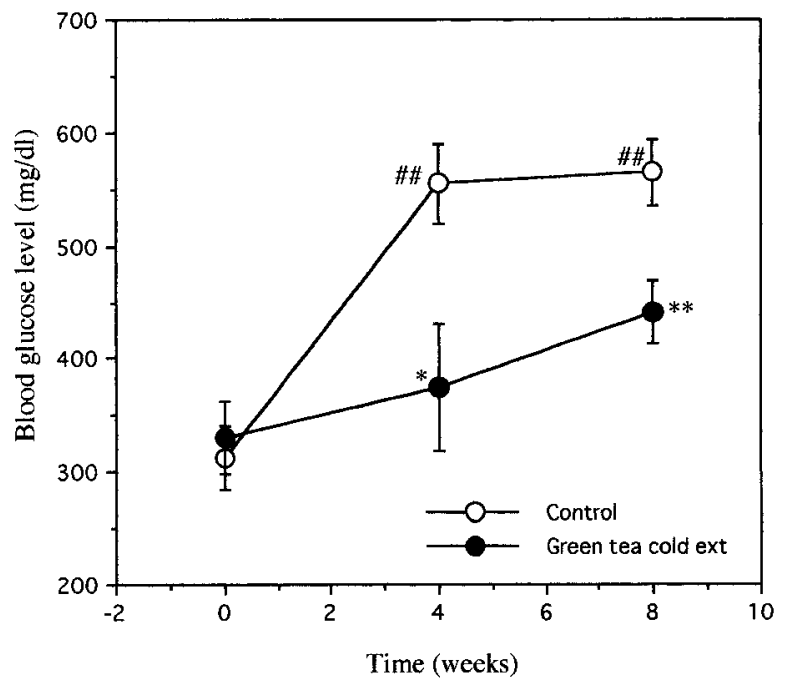

Fig. 1. Effects of Green Tea Cold Ext on Blood Glucose Levels in KK-Ay Mice (Repeated Administrations)

Green tea cold ext $100 \mathrm{mg} / \mathrm{kg}$ was administered orally to KK-Ay diabetic mice. After 4 and 8 weeks, blood samples were taken to determine blood glucose levels. Each value represents the mean \pm S.E. of 3-5 mice. Significantly different from pre administration value, $\# \# p<0.01$. Significantly different from control, ${ }^{*} p<0.05,{ }^{*} p<<0.01$.

subcutaneously. Blood samples were collected before administration of the insulin and 30,60, and 120 min later.

Determination of Blood Glucose and Insulin

Blood glucose levels in the mice were determined using the glucose oxidase method, ${ }^{3)}$ and plasma insulin levels were measured using the double-antibody method. ${ }^{4}$ All the data are expressed as mean \pm S.E., and Student's $t$-test and analysis of variance (ANOVA) are used for statistical analysis. The values were considered to differ significantly when the $p$-value was less than 0.05 .

\section{RESULTS}

The effects of the repeated administration of green tea cold ext on blood glucose levels are shown in Fig. 1. Green tea cold ext-treated $(100 \mathrm{mg} / \mathrm{kg})$ animals showed lower blood glucose levels from 4 to 8 weeks after the administration compared with the controls ( 4 weeks $p<0.05,8$ weeks $p<0.01$ ). The plasma insulin level in green tea cold ext-treated KK-Ay mice tended to decrease 8 weeks after administration (Fig. 2). However, green tea cold ext did not affect blood glucose levels in normal mice (Fig. 3).

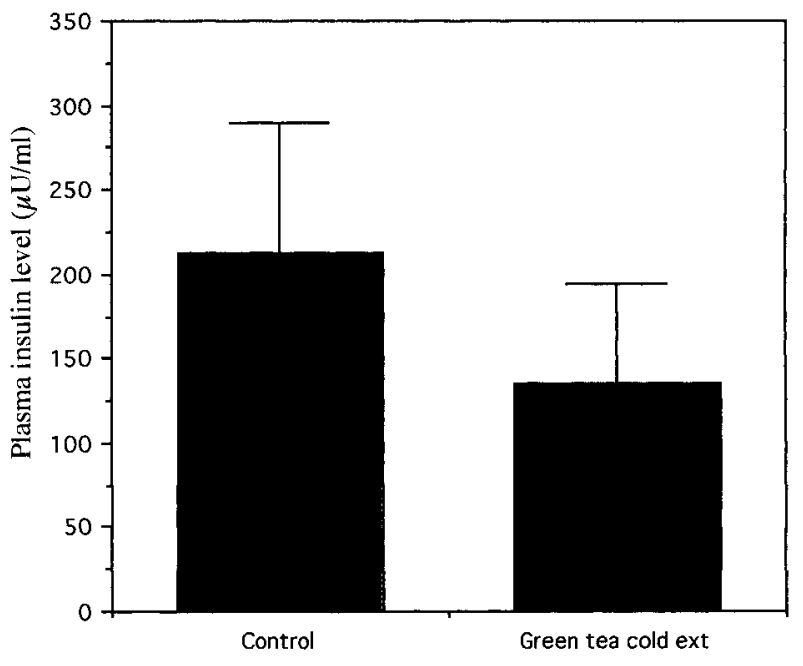

Fig. 2. Effects of Green Tea Cold Ext on Plasma Insulin Levels in KK-Ay Mice

Green tea cold ext $100 \mathrm{mg} / \mathrm{kg}$ was administered orally to KK-Ay diabetic mice. After 8 weeks, blood samples were taken to determine plasma insulin levels. Each value represents the mean \pm S.E. of 3-5 mice.

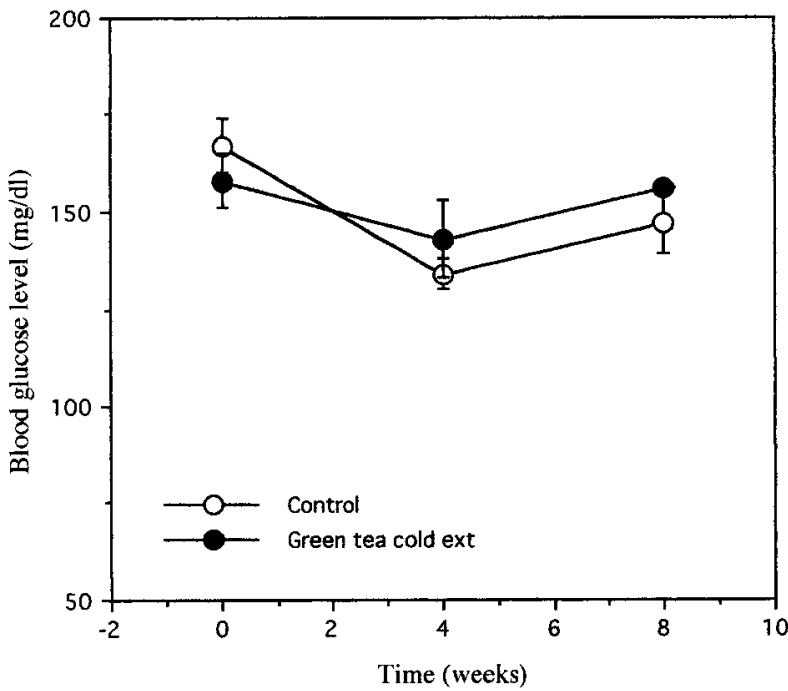

Fig. 3. Effects of Green Tea Cold Ext on Blood Glucose Levels in Normal Mice

Green tea cold ext $100 \mathrm{mg} / \mathrm{kg}$ was administered orally to normal $\mathrm{ddY}$ mice. After 4 and 8 weeks, blood samples were taken to determine blood glucose levels. Each value represents the mean \pm S.E. of 5 mice.

\section{Insulin Tolerance Test}

Green tea cold ext (100 mg/kg body weight p.o.) decreased blood glucose levels after $120 \mathrm{~min}$ compared with the controls $(p<0.05)$ in the insulin tolerance test (Fig. 4). 


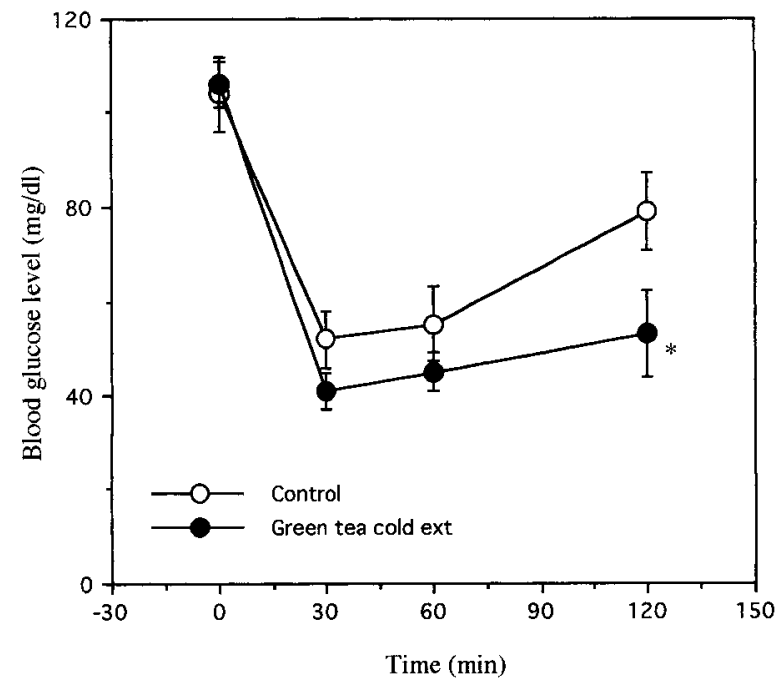

Fig. 4. Effects of Green Tea Cold Ext on Insulin Tolerance in KK-Ay Mice

After overnight fasting, insulin solution ( $0.5 \mathrm{U} / \mathrm{kg}$ body weight) was administered subcutaneously. Blood samples were collected before the administration of insulin and 30,60 and 120 min thereafter. Each value represents the mean \pm S.E. of 6-11 mice. *Significantly different from controls, $p<0.05$.

\section{DISCUSSION}

This study clearly showed that the water extract of green tea produces a consistent hypoglycemic effect. In a preliminary study, we examined the dose dependence $(20,100,300 \mathrm{mg} / \mathrm{kg})$ after oral administration of green tea and found that it showed hypoglycemic activity on maltose tolerance at 100 and $300 \mathrm{mg} / \mathrm{kg}$ (data not shown). Therefore we studied the effects of green tea at the dosage of $100 \mathrm{mg} / \mathrm{kg}$.

KK-Ay mice, an animal model of type 2 diabetes mellitus, are known for genetically induced diabetes and include ob/ob mice ${ }^{5)}$ and KK mice, ${ }^{6}$ which have hyperinsulinemia as a result of insulin resistance. ${ }^{7)}$ The blood glucose levels of control KK-Ay mice were elevated at 4 and 8 weeks when compared with 0 week. The KK-Ay mice used showed earlystage symptoms of type 2 diabetes. Green tea cold ext-treated KK-Ay mice showed lower blood glucose levels over the period from 4 to 8 weeks after administration. For early-stage symptoms of type 2 diabetes, diet and exercise therapy are used. These results indicate that green tea cold ext is useful in the treatment of type 2 diabetes.

Green tea cold ext also lowered blood glucose levels in the insulin tolerance test and hyper- insulinemia tended to improve. Insulin $(0.5 \mathrm{U} / \mathrm{kg})$ treated KK-Ay mice did not have lower blood glucose levels because of insulin resistance in the peripheral tissues, indicating that green tea cold ext lessens this resistance. Recently, Wu et al. have reported that green tea supplementation for 12 weeks ameliorates insulin resistance and increase glucose transporter IV content in a fructose-fed rat model. ${ }^{8)}$ Insulin resistance in peripheral tissues is known to be one of the pathogenic factors in type 2 diabetes, together with the impairment of glucose-induced insulin secretion from pancreatic beta cells. Therefore it is important that green tea cold ext improves insulin resistance.

Further studies should be performed to confirm that green tea cold ext could become useful in the treatment of diabetes through its unique therapeutic mechanism. The above experimental results suggest that the antidiabetic activity of green tea cold ext supports the traditional medical treatment of type 2 diabetes.

\section{REFERENCES}

1) Crespy, V. and Williamson, G. (2004) A review of the health effects of green tea catechins in in vivo animal models. J. Nutr, 134, 3431S-3440S.

2) Shimizu, M., Wada, S., Hayashi, T., Arisawa, M., Ikegaya, K., Ogaku, S., Yano, S. and Morita, N. (1988) Studies on hypoglycemic constituents of Japanese tea. Yakugaku Zasshi, 108, 964-970.

3) Marks, V. and Lloyd, K. (1963) Preservation of blood glucose analysis by glucose oxidase. Clin. Chim. Acta, 8, 326.

4) Bailei, C. J. and Ahmed-Sorour, H. (1980) Role of ovarian hormones in the long-term control of glucose homeostasis. Diabetologia, 19, 475-481.

5) Coleman, D. L. (1982) Diabetes-obesity syndromes in mice. Diabetes, 31(Suppl. 1), 1-6.

6) Nakamura, M. (1962) A diabetic strain of the mouse. Proc. Jpn. Acad. Sci., 38, 348-352.

7) Nishimura, M. (1969) Breeding of mice strains for diabetes mellitus. Exp. Anim., 18, 147-157.

8) Wu, L. Y., Juan, C. C., Hwang, L. S., Hsu, Y. P., Ho, P. H. and Ho, L. T. (2004) Green tea supplementation ameliorates insulin resistance and increases glucose transporter IV content in a fructose-fed rat model. Eur. J. Nutr., 43, 116-124. 